\title{
A Case Report on Life Threatening Hemorrhage in Preterm Pregnancy with Placenta Praevia and Management Challenges in a Low Neonatal Resourced Regional Hospital $187 \mathrm{~km}$ away from a Tertiary Center
}

\author{
Ayesha Akhter ${ }^{1, *}$, Bruno Giorgio ${ }^{1}$, Adebayo Adeyemi ${ }^{1}$, Md Rafiqul Islam ${ }^{1,2,3,4}$ \\ ${ }^{1}$ Goulburn Valley Health, Australia \\ ${ }^{2}$ Department of Rural Health, the University of Melbourne, Australia \\ ${ }^{3}$ School of Health and Social Development, Deakin University, Australia \\ ${ }^{4}$ Rumbalara Aboriginal Cooperative Limited, Australia
}

Copyright $\mathrm{O} 2017$ by authors, all rights reserved. Authors agree that this article remains permanently open access under the terms of the Creative Commons Attribution License 4.0 International License

\begin{abstract}
Placenta praevia is one of the obstetric emergencies, which can cause life threatening hemorrhage. Time interval to access support for these patients from emergency retrieval team are often challenging. Also, dilemmas may appear on taking decision for in-utero versus ex-utero transfer of neonates to tertiary hospital. In this case study, the patient presented with a life threatening antepartum hemorrhage (APH) in a low neonatal resourced regional hospital at 29 weeks of gestation. She was extremely needle phobic that delayed the resuscitation process. The overall situation and the patient's clinical condition were inappropriate for road transfer. Moreover, considering the weather condition on the day, air ambulance was grounded. Eventually, in-utero transfer of the neonate to tertiary hospital was impossible. As a result, she was delivered by an uncomplicated caesarean section and an initial resuscitation of the newborn was provided by the pediatric team of the hospital. Emergency neonatal retrieval team was able to arrive 3 hours after the birth for transferring the premature neonate to a tertiary center.
\end{abstract}

Keywords Life Threatening APH, Low Neonatal Resourced Hospital, Transfer Difficulties, Decision Dilemmas

\section{Introduction}

Antepartum hemorrhage (APH) is defined as bleeding from or into the genital tract, occurring from $24+0$ weeks of pregnancy and prior to the birth of the newborn [1]. APH associated with maternal or fetal compromise is an obstetric emergency. A low lying placenta occurs in $5 \%$ of pregnancies at 16-18 weeks gestation but is evident in only $0.5 \%$ pregnancies at term [2]. Placenta praevia accounts for $30 \%$ of antepartum hemorrhages and is a leading cause of perinatal and maternal mortality worldwide [3]. Khan KS et al (2006) reported that APH causes up to $50 \%$ of the estimated 500,000 maternal deaths that occur globally in each year [4]. Management should include maternal resuscitation and delivery of the fetus to control the bleeding [2]. Placenta praevia comprises a major role in causing preterm deliveries. Preterm babies are vulnerable to severe complications including neonatal death. It is, therefore, essential to provide timely adequate support to prevent short term and long term complications of preterm newborns. Preterm babies born in low neonatal resourced facilities need to transfer to well-equipped tertiary centers which can be sometimes challenging thus warranted an onsite management. Therefore, it is critical to offer continuing adequate training and skills for the staff of these low resourced, regional and remote hospitals that provide initial supports to such preterm newborns. We report a case of placenta praevia here with several aspects of management challenges in a regional Australian hospital. We obtained written informed consent from the patient to disseminate and publish the case anonymously.

\section{The Case}

In this case report, the patient was 22 years of old, non-indigenous, Australian born pregnant woman with one prior normal vaginal delivery. On admission, her BMI was 27. The morphology ultrasound at 19 weeks revealed anterior low-lying placenta two centimeters from the internal os. She presented approximately at $11 \mathrm{am}$ in a low neonatal 
resourced regional hospital at 29 weeks of pregnancy with mild per vaginal bleeding. She was assessed as cervical bleeding in speculum examination and discharged home in the afternoon of the same day. On the following day at 3 am, she presented to the hospital again with painless unprovoked per vaginal bleeding and lost about $700 \mathrm{ml}$ of blood at home. On admission, her blood pressure was $140 / 80 \mathrm{mmHg}$ and heart rate was $110 / \mathrm{min}$. The uterus was soft and non-tender, cephalic presentation with reactive CTG. Speculum examination confirmed moderate vaginal bleeding with clot. After analyzing all the information, a plan was made to resuscitate with intravenous fluid, insertion of two wide-bore intravenous cannulas, blood cross-match, intramuscular betamethasone for the fetus, and transfer to a tertiary hospital at an expedited way. Emergency retrieval team was contacted and a bed was organized in a distant tertiary hospital. Road transfer was the only option as air ambulance was grounded for unfavorable weather. However, the patient declined both intravenous and intramuscular needle access as she was extremely needle phobic. Therefore ambulance staff denied transferring the patient without resuscitating the patient primarily. Subsequently, it took a long time to take consent for needle insertion after counselling from the patient at that life threatening condition. By the time she consented, there was a loss of two liters of blood in total; heart rate was increased and continued bleeding actively. Despite all the above difficulties and at this critical stage, the decision was made to delivering the baby immediately by cesarean section in that low neonatal resourced regional hospital after securing support from the emergency retrieval team. Simultaneously, the woman was resuscitated with blood transfusion and crystalloid solution. Immediately after this, a $1.3 \mathrm{~kg}$ baby girl was delivered by uncomplicated cesarean section under general anesthesia. The APGAR score of the newborn was 5 in one minute and 7 in five minutes. The baby was wrapped in a plastic bag, intubation done, CPAP, IPPV performed and transferred to the special care nursery of the hospital which had a capacity to support newborn babies after 32 weeks. Emergency retrieval team became available three hours after the delivery and the newborn was then transferred to a tertiary care center. The mother remained in the regional hospital ICU and the recovery was uneventful.

\section{Discussion}

Hemorrhage emerges as the major cause of severe maternal morbidity in almost all 'near miss' audits in both developed and developing countries [5]. Since placenta praevia is one of the leading causes of maternal hemorrhage, it is very important to diagnose as early as possible. Placenta praevia refers to presence of placental tissue at the lower uterine segment that partially or completely covers the cervical os. It is classified by ultrasound appearance that helps to understand what is clinically relevant. If placenta covers cervical os then it is called major placenta praevia and when it lies on the lower segment without covering the cervix is called minor placenta praevia. Routine morphology scan at 20 weeks of gestation usually can suggest placenta praevia. In case of minor placenta previa further ultrasound at around 32 weeks of gestation is recommended, because with the growth of the uterus over the time of pregnancy, the lower segment can extend and lead to increase the distance of placenta from cervix. Owing to the development of the lower uterine segment, placental 'apparent' migration occurs during the second and third trimesters [6] but for posterior placenta it is less likely to occur [7] or if there has been a previous caesarean section. However, most evident complication of placenta praevia is bleeding at rate of mild, moderate to severe. Usually bleeding is painless, recurrent, can be provoked by sexual intercourse. Once the diagnosis is made, it is important to counsel the patient regarding the possible complications and to inform immediate management including insertion of IV (intravenous) cannula needs to be done for the safety of the patient herself as well as for the fetus. It would be appropriate for certain cases, such as, this patient, to refer to a counselor to alleviate needle phobia as the most common complication of placenta previa is bleeding and volume replacement via IV cannula is critical. Patient with severe needle phobia should be informed about the consequences and make a practical approach for intravenous access during emergency situation. This approach will help reducing unnecessary delay during emergency situation. In this particular case, intravenous access was very important. Despite the patient was informed regarding possible disasters during that emergency situation, she was extremely adamant and did not let the health care providers to perform an IV access. Different approach was made by different clinicians to insert at least one IV access, nevertheless, it was declined by the patient and during this counseling process patient was continuing to loose blood. Since the primary resuscitation was not possible at the initial stage, therefore, road transfer was considered unsafe and the whole process was delayed further. Later, assistance was sought from anaesthetic colleague and after another half an hour with the help of Entonox, IV access was sited. Immediate resuscitation was performed with packed cell and isotonic solution. In the mean time it was already too late to transfer this patient safely to a tertiary center, which was about two hours away from that regional setting. Eventually decision was made to deliver twenty-nine week fetus in that level two neonatal hospitals. The possible outcome of the neonate was informed to the patient and the patient was counseled with adequate information such as the risk of complications increases with premature delivery of the baby. Prematurity and low birth weight (LBW) are major contributors to infant morbidity and mortality [8,9]. According to the Eunice Kennedy Shriver National Institute of Child Health and Human Development (NICHD) Neonatal Research Network, increased frequencies of multiple complications can be seen in very low birth weight (VLBW) infants [10], such as respiratory distress (93\%), 
retinopathy of prematurity $(59 \%)$, patent ductus arteriosus $(46 \%)$, bronchopulmonary dysplasia $(42 \%)$, late-onset sepsis(36\%), necrotizing enterocolitis (11\%), grade III intraventricular hemorrhage (IVH) and Grade IV IVH (7\% and $9 \%$, respectively), and periventricular leukomalacia (3\%). Other common complications include hypothermia, hypoglycaemia, low blood pressure and infection with many other long term complications. To prevent and to reduce the severity of the complication prompt and appropriate measures are imperative. Patient with imminent preterm delivery presenting in the low neonatal resourced hospital it is vital to transfer to a high level care. The real world scenario in this case was the new born delivered by emergency caesarean section and the on call paediatric team received the newborn in a plastic bag, and continued resuscitation. The neonatal retrieval team arrived few hours after the delivery. The newborn then transferred to the tertiary center for further management. These required to be happened in a faster and quicker way followed by an appropriate decision taking mechanism for saving maternal and foetal lives.

However, considering multiple contributing factors, in utero transfer is not always possible. Therefore, for the safety of the mother and baby, adequate support from local staff must play a vital role. To maintain suitable outcomes in such cases in the low resourced hospital, regular training and simulation for the staffs are crucial. Furthermore, antenatal counseling can play a vital role to avoid any obstetric disaster.

\section{Competing Interest}

The authors declare no competing interest.

\section{Authors Contribution}

AA conceptualized the idea and wrote the article. BG and
AB: conceptualized and supervised. MRI: mentored, revised and wrote the article.

\section{REFERENCES}

[1] RCOG Green-top Guideline No. 63

[2] Neilson JP. Interventions for suspected placenta praevia. Cochrane Database of Systematic Reviews 2003, Issue 2.

[3] Calleja-Agius J, Custo R, Brincat MP, Calleja N. Placental abruption and placenta praevia. Eur Clin Obstet Gynaecol 2006; 2: 121-7.

[4] Khan KS, Wojdyla D, Say L, Gülmezoglu AM, Van Look PF. WHO analysis of causes of maternal death: a systematic review. Lancet 2006; 367: 1066-74.

[5] Brace V, Kernaghan D, Penney G. Learning from adverse clinical outcomes: major obstetric hemorrhage in Scotland, 2003-05.BJOG 2007; 114: 1388-1396.

[6] Becker RH, Vonk R, Mende BC, Ragosch V, Entezami M. Therelevance of placental location at 20-23 gestational weeks for prediction of placenta previa at delivery: evaluation of 8650

[7] Ghourab S, Al-Jabari A. Placental migration and mode of delivery in placenta previa: transvaginal sonographic assessment during the third trimester. Ann Saudi Med 2000; 20: $382-5$.

[8] Mathews TJ, MacDorman MF and Thoma ME. Infant mortality statistics from the 2013 period linked birth/infant death data set. Natl Vital Stat Rep 2015; 64:1.

[9] Watkins WJ, Kotecha SJ, Kotecha S. All-Cause Mortality of Low Birthweight Infants in Infancy, Childhood, and Adolescence: Population Study of England and Wales. PLoS Med 2016; 13:e1002018

[10] Stoll BJ, Hansen NI, Bell EF, et al. Neonatal outcomes of extremely preterm infants from the NICHD Neonatal Research Network. Pediatrics 2010; 126:443. 